\title{
On Uniform Resampling and Gaze Analysis of Bidirectional Texture Functions
}

\author{
JIŘí FILIP ${ }^{1,2}$, MICHAEL J. CHANTLER ${ }^{1}$, MICHAL HAINDL $^{2}$ \\ Heriot-Watt University ${ }^{1}$ \\ Institute of Information Theory and Automation of the AS CR ${ }^{2}$
}

\begin{abstract}
The use of illumination and view dependent texture information is recently the best way to capture the appearance of real-world materials accurately. One example is the Bidirectional Texture Function. The main disadvantage of these data is their massive size. In this paper we employ perceptually-based methods to allow more efficient handling of these data. In the first step we analyse different uniform resampling by means of a psychophysical study with eleven subjects, comparing original data with rendering of a uniformly resampled version over the hemisphere of illumination and view dependent textural measurements. We have found that down-sampling in view and illumination azimuthal angles is less apparent than in elevation angles and that illumination directions can be down-sampled more than view directions without loss of visual accuracy. In the second step we analysed subjects gaze fixation during the experiment. The gaze analysis confirmed resuls from the experiment and revealed that subjects were fixating at locations aligned with direction of main gradient in rendered stimuli. As this gradient was mostly aligned with illumination gradient we conclude that subjects were observing materials mainly in direction of illumination gradient. Our results provide interesting insights in human perception of real materials and show promising consequences for development of more efficient compression and rendering algorithms using these kind of massive data.
\end{abstract}

Categories and Subject Descriptors: I.3.7 [Three-Dimensional Graphics and Realism]: Color, shading, shadowing, and texture; I.4.7 [Image Processing and Computer Vision]: Feature Measurement-Texture; J.4 [Social and Behavioral Sciences]: Psychology

General Terms: Human Factors, Experimentation

Additional Key Words and Phrases: BTF, uniform resampling, visual degradation, phychophysical experiment, texture compression, eye-tracking

\section{INTRODUCTION}

In many industrial sectors, demand is currently increasing for accurate virtual representation of real-world materials. Important application areas include among others safety simulations and computer-aided design. In the former area, the main concern is choosing the right material to fulfill given safety limits of reflectance, while in the later the aim is to avoid costly and time consuming design cycles of material selection, solid model production and visual evaluation. These tasks, among others, require accurate photo-realistic representations of real material samples dependent

Authors contact address: filipj@utia.cas.cz

Permission to make digital/hard copy of all or part of this material without fee for personal or classroom use provided that the copies are not made or distributed for profit or commercial advantage, the ACM copyright/server notice, the title of the publication, and its date appear, and notice is given that copying is by permission of the ACM, Inc. To copy otherwise, to republish, to post on servers, or to redistribute to lists requires prior specific permission and/or a fee.

(c) $20 \mathrm{YY}$ ACM 0000-0000/20YY/0000-0001 $\$ 5.00$ 


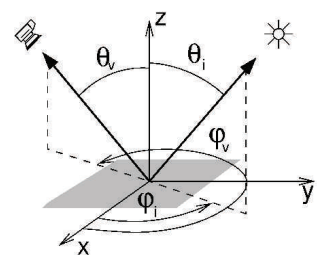

Fig. 1: Relationship between illumination and viewing angles within texture coordinate space.

on different illumination and viewing conditions.

One such representation is the Bidirectional Reflectance Distribution Function [Nicodemus et al. 1977] (BRDF). The BRDF is defined as ratio of radiance reflected from the material (outgoing) to the illuminating radiance (incoming) for all possible pairs of incoming $\omega_{i}\left(\theta_{i}, \phi_{i}\right)$ and outgoing $\omega_{v}\left(\theta_{v}, \phi_{v}\right)$ directions (see Fig. 1). This ratio is spectral dependent ( $d$ spectral channel) and results in a five-dimensional multispectral function $B R D F_{d}\left(\theta_{i}, \phi_{i}, \theta_{v}, \phi_{v}\right)$. This function possesses two important properties; energy conservation and reciprocity of incoming and outgoing directions. As the BRDF captures the reflectance of only a small or averaged portion of the material surface, it is most applicable to surfaces without texture such as paints and similar finishes. Although spatially varying BRDFs can be used to describe textured materials [Pellacini and Lawrence 2007], it is limited by its properties to smooth and opaque surfaces. The first real illumination/view dependent surface texture representation was the Bidirectional Texture Function (BTF), introduced in [Dana et al. 1999]. A BTF is a six-dimensional function representing the appearance of a sample surface for variable illumination and view directions. Compared to a five-dimensional BRDF, a BTF depends on two additional spatial parameters, a planar position $(x, y)$ over a material surface, resulting in seven-dimensional multispectral function $B T F_{d}\left(x, y, \theta_{i}, \phi_{i}, \theta_{v}, \phi_{v}\right)$.

The BTF represents such effects as masking, shadowing, inter-reflections and sub-surface scattering. During recent years, different BTF measurement systems have appeared, based on different principles each offering different advantages and disadvantages. Although material visualization using BTFs provide superb visual quality, even an average BTF sample (e.g., 256x256) often reaches gigabytes in size. This data size can be edited almost interactively by careful data management and empirical editing operators [Kautz et al. 2007]. However, it is still beyond the real-time rendering capabilities of current graphics hardware, and so there have been many recent research attempts to develop an efficient compression techniques that allow computationally cheap reconstruction and visualization of BTFs (recent surveys [Müller et al. 2005] and [Filip and Haindl 2009]). All such methods compress a full BTF sample, which often leads to extreme computational and excessive storage demands.

In this paper we aim to analyse impact of several uniform resampling schemes on visual quality of images rendered from resampled BTF data. The visual quality is being assessed by human observers. To achieve this we propose a psychophysical study of various uniform BTF resampling schemes performed on eight datasets. This paper starts with recapitulation of published work in this research area in Section 2. Section 3 describes the experimental dataset and proposes several reACM Transactions on Applied Perception, Vol. V, No. N, Month 20 YY. 


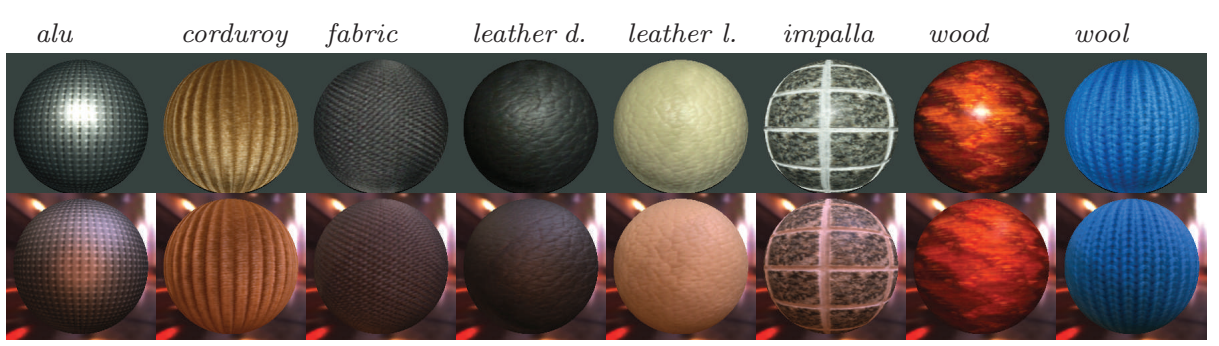

Fig. 2: Examples of used BTF samples illuminated by point-light and environment illumination.

sampling schemes applied on these data. Design of the proposed psychological experiment is in Section 4. Discussion over the obtained results from subjects' responses and their gaze fixation analysis are in Section 5. Section 6 summarizes and concludes the paper.

\section{PRIOR WORK}

\subsection{Psychovisual Analysis}

To the best of our knowledge, there are few publications on psychophysical analysis of view and illumination dependent texture data. Several papers investigate influences of light position, material reflectance, view position, or surface shape [Fleming et al. 2003], [Lawson et al. 2003], [te Pas and Pont 2005a], [te Pas and Pont 2005b], [Ramanarayanan et al. 2007]. [Pellacini et al. 2000] derived a psychophysically-based model of light reflection with two perceptually meaningful uniform dimensions. [Matusik et al. 2003] performed psychophysical tests showing consistent transitions in perceived properties between different BRDFs. Meseth et al [Meseth et al. 2006] shows a study comparing performance of material photographs, BTF rendering, and flat textures modulated by BRDFs for the same illumination condition. Different BTF compression and modelling methods are perceptually compared in a recent survey [Filip and Haindl 2009]. Methods mentioned above investigate influences of light, view, material reflectance, or shape. The only method dealing with optimal sparse sampling of view/illumination dependent textural data was introduced in [Filip et al. 2008]. This method is based on a psychophysical study and enables significant reduction of BTF images, while still providing the same visual quality. In comparison with the previous research this paper psychophysically assesses different uniform resampling of BTF data.

\subsection{Eye-Gaze Analysis}

Eye-tracking methods has been an important source of information about human visual perception. Their typical application is visual search where a subject's task is to identify presence or magnitude of specific features in stimulus image [Pomplun 2006], [Over et al. 2007]. Gaze analysis has allowed the development of many applications [Duchowski 2002], e.g., gaze-contingent displays [Duchowski et al. 2004], methods for eye motion synthesis [Lee et al. 2002], [Deng et al. 2005] or for prediction of fixation behavior in computer games [Sundstedt et al. 2008]. Although Elhelw et al. [Elhelw et al. 2008] used eye tracking to analyse visual realism 
in simulated medical scenes, we are not aware of any relevant research analysing human gaze behavior for stimuli representing realistic materials' properties captured by bidirectional texture functions.

\section{PROPOSED DATA RESAMPLING}

In this paper we have chosen BTF data as a typical example of simultaneous illumination and view dependent data. We have used the datasets from the Bonn BTF database $^{1}$. For reduction of the size of processed datasets and simultaneously for enabling seamless covering of the test object, a BTF data tiling approach was applied. We have chosen BTF datasets corresponding to distinct types of real-world materials. Thus the following six different BTF datasets formed the subject of our experiment: aluminum profile (alu), corduroy fabric (corduroy), dark cushion fabric (fabric), artificial dark leather (leather d.), artificial light leather (leather l.), glazed tile with white pointing (impalla), lacquered wood (wood), and knitted wool (wool). Examples of these materials for both tested illumination environments are shown in Fig. 2. The original datasets have an angular resolution of illumination and viewing angles of $n_{i} \times n_{v}=81 \times 81$ (see Fig. 3-left).

In order to obtain considerable reduction of BTF dataset size we adopted three different BTF sampling schemes denoted as A, B, and $\mathbf{C}$ in Fig. 3. Each of the

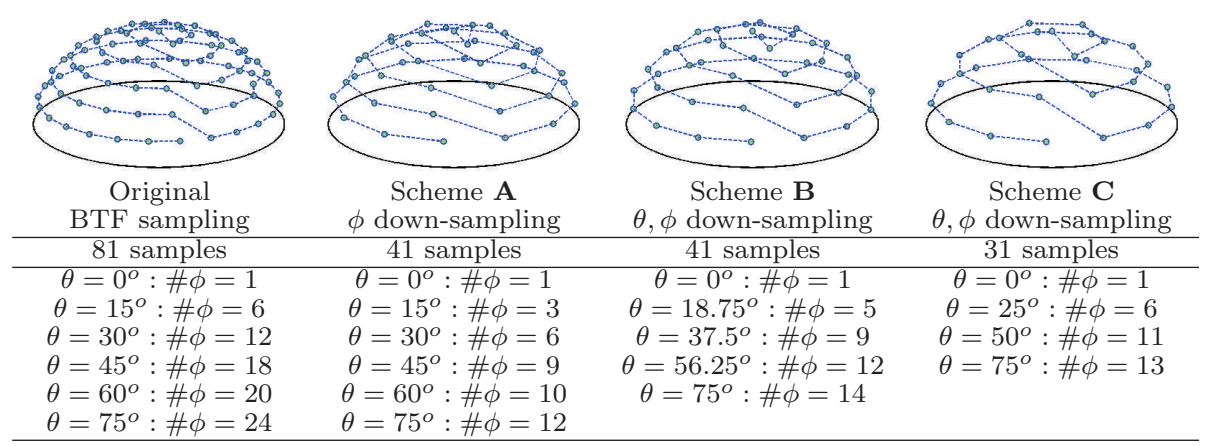

Fig. 3: Sampling of original BTF measurements (left) compared with its three tested resampled schemes: $\boldsymbol{A}$ - along azimuth $\theta, \boldsymbol{B}, \boldsymbol{C}$ - along azimuth $\theta$ and elevation $\phi$ angles. At the bottom are numbers of azimuthal samples for each elevation level.

schemes is designed to fulfill uniform sampling in azimuthal angle $\phi$. While scheme A preserves original sampling of elevation angle $\theta$ but reduces the number of azimuthal samples along angle $\phi$, schemes $\mathbf{B}$ and $\mathbf{C}$ reduce sampling for both angles. While schemes $\mathbf{A}$ and $\mathbf{B}$ produce the same number of samples, i.e., 41, scheme $\mathbf{C}$ reduces the number of samples even more aggressively yielding only 31 samples. Numbers of samples for individual levels of elevation angle $\theta$ for individual resampling schemes are given at the bottom of Fig. 3. Note that the view and illumination dependent data, i.e., BTF, require directional sampling of both illumination $\omega_{i}\left(\theta_{v}, \phi_{v}\right)$ and view directions $\omega_{v}\left(\theta_{v}, \phi_{v}\right)$. However, in these two directions

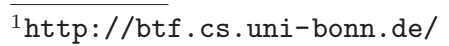

ACM Transactions on Applied Perception, Vol. V, No. N, Month 20 YY. 


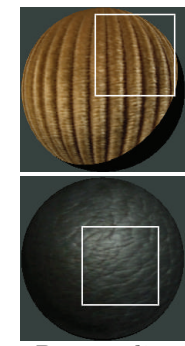

Resampling: $(\mathrm{I} \times \mathrm{V})$ Images:

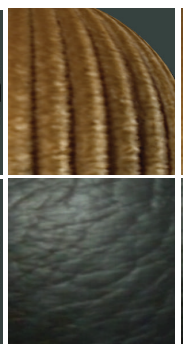

original $81 \times 81$

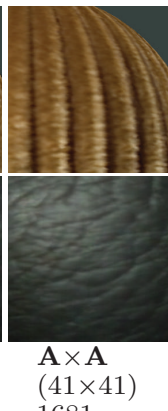

1681

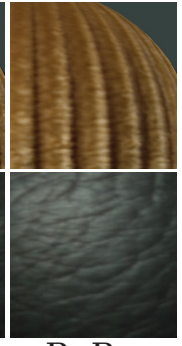

$\mathbf{B} \times \mathbf{B}$

$(41 \times 41)$ 1681

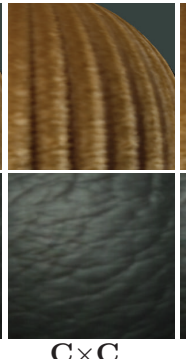

961

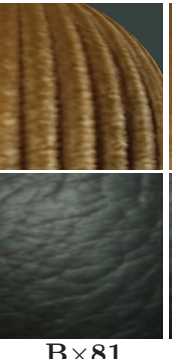

$(41 \times 81)$

3321

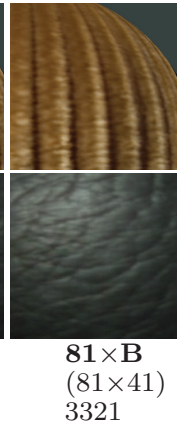

Fig. 4: Examples of corduroy and leather d. BTF samples with illumination/view directions uniformly resampled in the five proposed ways.

we can adopt different sampling schemes without limiting practical usage of the data. Thus we decided to resample the original BTF datasets in five different test sets. The first three are straightforward and resample both $\omega_{i} \times \omega_{v}$ directions in the same way, using a combination of the same schemes $\mathbf{A} \times \mathbf{A}, \mathbf{B} \times \mathbf{B}$, and $\mathbf{C} \times \mathbf{C}$. The last two, used resample scheme $\mathbf{B}$ on either $\omega_{i}$ or on $\omega_{v}$. This resulted in resampling patterns of $\mathbf{B} \times \mathbf{8 1}$ and $81 \times \mathbf{B}$. Consequently, the resampled datasets use the following numbers of BTF images:
$\mathbf{A} \times \mathbf{A} \quad 1681$ images $(26 \%)$
$\mathbf{B} \times \mathbf{B} \quad 1681$ images $(26 \%)$
$\mathbf{C} \times \mathbf{C} \quad 961$ images $(15 \%)$
$\mathbf{B} \times \mathbf{8 1} 3321$ images $(51 \%)$
$\mathbf{8 1} \times \mathbf{B} 3321$ images $(51 \%)$.

Note that the original number of images in each dataset is 6561 . To avoid introduction of local errors into the original data by means of their down-sampling using local interpolation we used a two-step global interpolation scheme based on radialbasis functions [Carr et al. 2001]. In the first step the data for all illumination directions $\omega_{i}$ for fixed viewing direction $\omega_{v}$ are interpolated into a new illumination discretization scheme and these interpolated values for all combinations of $\theta_{i}$ and $\phi_{i}$ angles are further interpolated into a new viewing direction discretization scheme. Finally, all eight datasets were resampled in the five proposed ways (see Fig. 4) and together with the original datasets used in the following psychophysical experiment.

\section{PSYCHOPHYSICAL EXPERIMENT}

The goal of the experiment was to analyze the influence of different illumination and view direction resampling schemes on the final appearance of rendered images.

Experimental Stimuli. As experimental stimuli we have used pairs of static images of size $800 \times 800$, representing a material BTF rendered on a 3D object. Each pair consisted of a rendering using the full original dataset and one using one of the five resampled datasets. Pairs of images were displayed simultaneously, side-by-side. A sphere was used as a test object rendered for point-light and grace $^{2}$ illumination environments. The point-light was positioned on the top-left from a viewing position consistent with the surrounding physical illumination. The en-

\footnotetext{
${ }^{2}$ http://www.debevec.org
} 
(a)

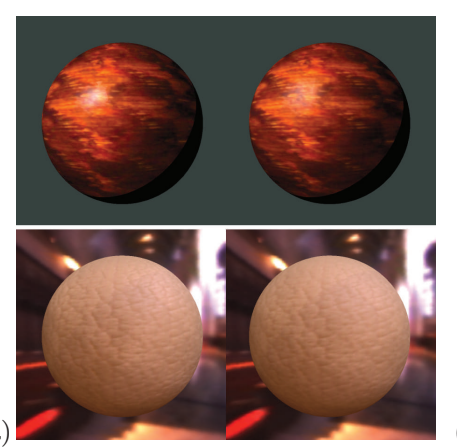

(b)

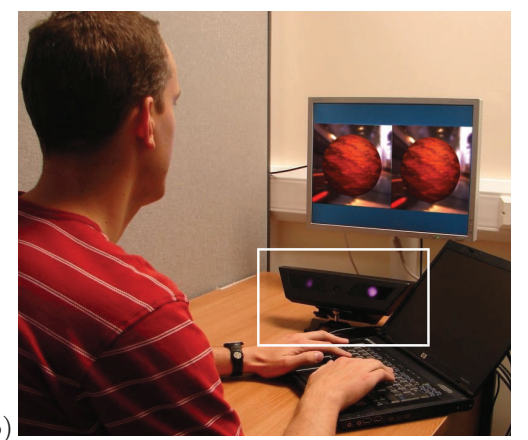

Fig. 5: Examples of two stimuli (a) and experimental setup (b) with the eye-tracker highlighted.

vironment maps were approximated by a set of 144 discrete point-lights [Havran et al. 2005]. The background of the point-light illuminated stimuli, and the remaining space on the screen, was set to dark gray. Example stimuli are shown in Fig. 5-a. Given eight material BTFs, five different resampling schemes proposed in Section 3 and two different illumination types, the total number of stimuli was 80 .

Participants. Eleven paid observers (six males, five females) participated in the experiments. All were students or university employees working in different fields, were less than 35 years of age, and had normal or corrected to normal vision. All were naive with respect to the purpose and design of the experiment.

Experimental Procedure. The participants were shown the 80 stimuli in a random order and asked a yes-no question: 'Can you detect any differences in the material covering the two spheres?'. There was a pause of two seconds between stimuli presentations, and participants took on average less than 40 minutes to perform the whole experiment. All stimuli were presented on a calibrated 20.1" NEC2090UXi LCD display $(60 \mathrm{~Hz}$, resolution $1600 \times 1200$, color temperature $6500 \mathrm{~K}$, gamma 2.2, luminance $\left.120 \mathrm{~cd} / \mathrm{m}^{2}\right)$. The experiment was performed under dim room lighting. Participants viewed the screen at a distance of $0.9 \mathrm{~m}$, so that each sphere in a pair subtended approximately $9^{\circ}$ of visual angle.

Observers gaze data were recorded using a Tobii x50 infrared-based binocular eye-tracking device as shown in Fig. 5-b. The device was calibrated for each subject individually and provided the locations and durations of fixations at a speed 50 samples/s. Maximum error specified by manufacturer is approximately $\pm 0.5^{\circ}$ of visual angle, which corresponds to \pm 32 pixels for our setup and stimuli resolution. The shortest fixation duration to be recorded was set to $100 \mathrm{~ms}$.

Experimental Results Analysis. When participants reported a difference between the rendered images their response was assigned a value of 1 , and otherwise 0. By averaging the responses of all participants, we obtained psychometric data for eight tested BTF samples, two different illumination schemes and the five proposed resampling schemes. The following section analyses and discusses the results of the experiment.

ACM Transactions on Applied Perception, Vol. V, No. N, Month 20 YY. 


\section{RESULTS AND DISCUSSION}

\subsection{Subjects' Responses Analysis}

Results of the experiment for all five test sets in are shown in Fig. 6 and Fig. 7. All graphs in the figures show perceptual values of observed differences between the renderings of original and resampled BTF data. Fig. 6 shows average participants' responses for resampling schemes $\mathbf{A} \times \mathbf{A}, \mathbf{B} \times \mathbf{B}$, and $\mathbf{C} \times \mathbf{C}$. Fig. 7 illustrates responses for resampling schemes $8 \mathbf{8 1} \times \mathbf{B}, \mathbf{B} \times \mathbf{8 1}$, and $\mathbf{B} \times \mathbf{B}$. Both figures show results for point-light (a) and grace environment illumination (b). The graphs include error-bars representing twice the standard error. Additionally, we performed Cochran Q-test [Cochran 1950] on the original dichotomous data obtained from the experiment. The obtained confidence intervals $\left(p_{Q}\right)$ corresponding to the tested datasets are shown below the individual graphs. Despite a relatively low number of tested subjects, we can see that for most of the datasets for point-light we have got quite significant values (the values fulfilling $75 \%$ significance test are underlined). The least significant values $\left(p_{Q}>0.2\right)$ were estimated for specular samples (alu and wood), where most of the resampling schemes blure specular highlights. The $p_{Q}$ values for grace environment are often less significant than for point-light, that is caused by less apparent differences between spheres in the stimuli.

In Fig. 6 we can observe a significant increase in perceived difference when resampling scheme $\mathbf{B} \times \mathbf{B}$ is used comparing to the scheme $\mathbf{A} \times \mathbf{A}$. This pattern was visible for both types of illumination. This means that the participants were much more sensitive to reduction of samples along elevation angle $\theta$ than to reduction of samples along azimuthal angle $\phi$. More aggressive down-sampling $\mathbf{C} \times \mathbf{C}$ did not introduce much more difference. Similar behavior can be found in Fig. 7, where resampling of view direction $81 \times \mathbf{B}$ introduces a significantly higher perceptual difference than resampling of illumination direction $\mathbf{B} \times \mathbf{8 1}$. When we compare resampling of view direction $81 \times \mathbf{B}$ with resampling of both direction $\mathbf{B} \times \mathbf{B}$ we cannot observe any particular increase in the perceptual difference. While the datasets of highly structured fabrics samples corduroy, fabric, and wool comply the most with the described behavior, the datasets corresponding to altogether smoother and more specular materials alu and wood have similar performance for point-light illumination regardless of the resampling scheme used. Fig. 6 suggests that using resampling based on scheme $\mathbf{A}$ can give even better visual performance, while using the same number of BTF images (3321 for $\mathbf{A} \times \mathbf{8 1}$ ). This allows considerable reduction of original $6561 \mathrm{BTF}$ images, that are used as input data in many compression and modeling algorithms, without any particular perceptual error (see Fig. 4). This conclusion holds mainly for environment illumination which is, however, the prevailing type of illumination used in contemporary rendering systems.

\subsection{Gaze Fixations Analysis}

Although the subjects' responses are an important source of psychophysical data they do not provide us with any spatial information concerning the underlying sample properties and their perception by the subjects. To overcome this, we recorded gaze fixations of all eleven subjects throughout the experiment. A rigorous analysis of such eye-tracking data revealed interesting insights on how subjects observed different materials when assessing the performance of the five proposed 


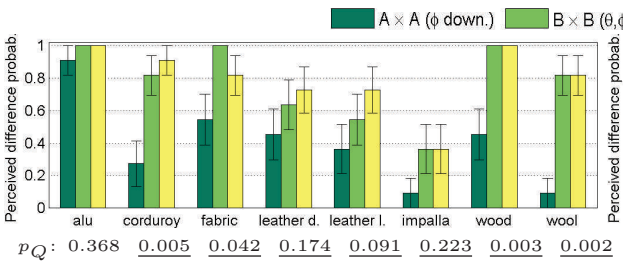

(a)

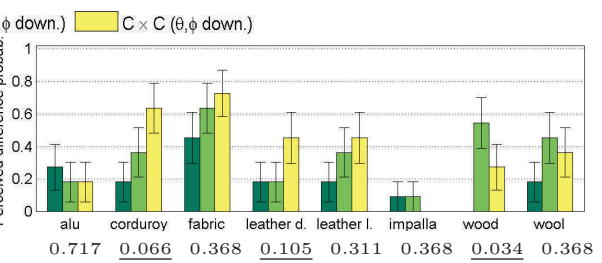

(b)

Fig. 6: Mean perceived difference for all tested resampling schemes $\boldsymbol{A} \times \boldsymbol{A}, \boldsymbol{B} \times \boldsymbol{B}$, and $\boldsymbol{C} \times \boldsymbol{C}$ for different datasets and (a) point-light, (b) grace illumination environment.

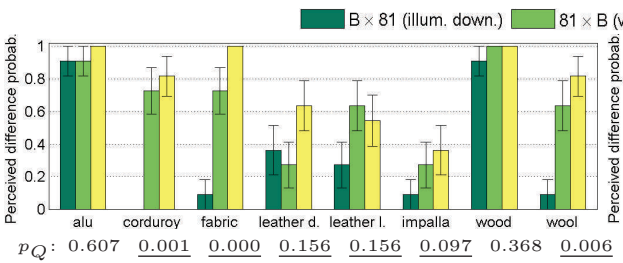

(a)

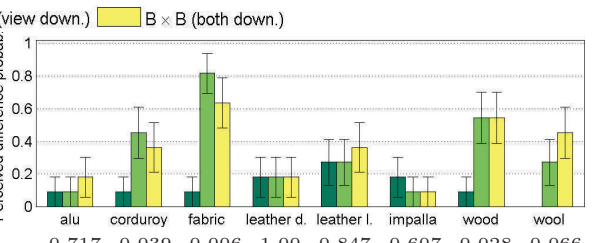

(b)

Fig. 7: Mean perceived difference for resampling of illumination/view/both respectively using the scheme $\boldsymbol{B}$ for different datasets and (a) point-light, (b) grace illumination environment.

resampling schemes.

During the experiment subjects performed a visual search task, looking for differences in texture on the spheres and thus they were consequently comparing similar spatial locations on both spheres. One of the spheres was always rendered using the original data while the other one was rendered using one of the proposed resampling schemes. In total we recorded over 9197 fixations for point light and 10327

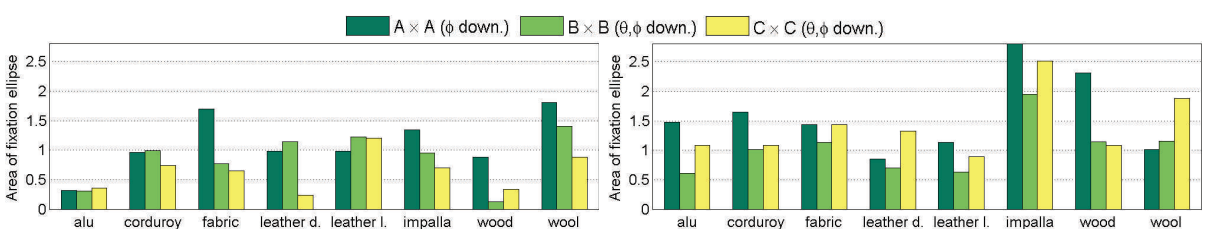

(a)

(b)

Fig. 8: Comparison of fixation areas for resampling schemes $\boldsymbol{A} \times \boldsymbol{A}, \boldsymbol{B} \times \boldsymbol{B}$, and $\boldsymbol{C} \times \boldsymbol{C}$ for different datasets and (a) point-light, (b) grace illumination environment.

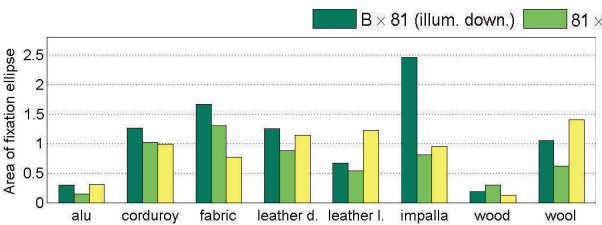

(a)

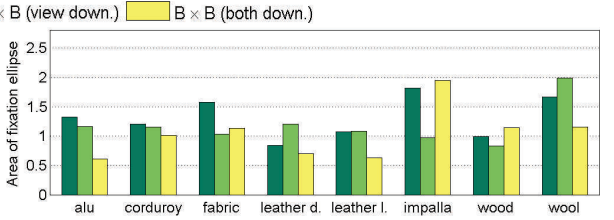

(b)

Fig. 9: Comparison of fixation areas for resampling of illumination/view/both respectively using the scheme $\boldsymbol{B}$ for different datasets and (a) point-light, (b) grace illumination environment.

ACM Transactions on Applied Perception, Vol. V, No. N, Month 20 YY. 
fixations for grace environment illumination. Only those fixations measured within the textured spheres were used in further analysis. Numbers of fixations and their spatial distribution for individual materials across all subjects for point-light and grace illumination environments are shown Fig. 10. As the placement of the spheres was vertically symmetrical and the horizontal position of original and resampled rendering was random we mirrored all fixations onto one sphere to ease further processing.

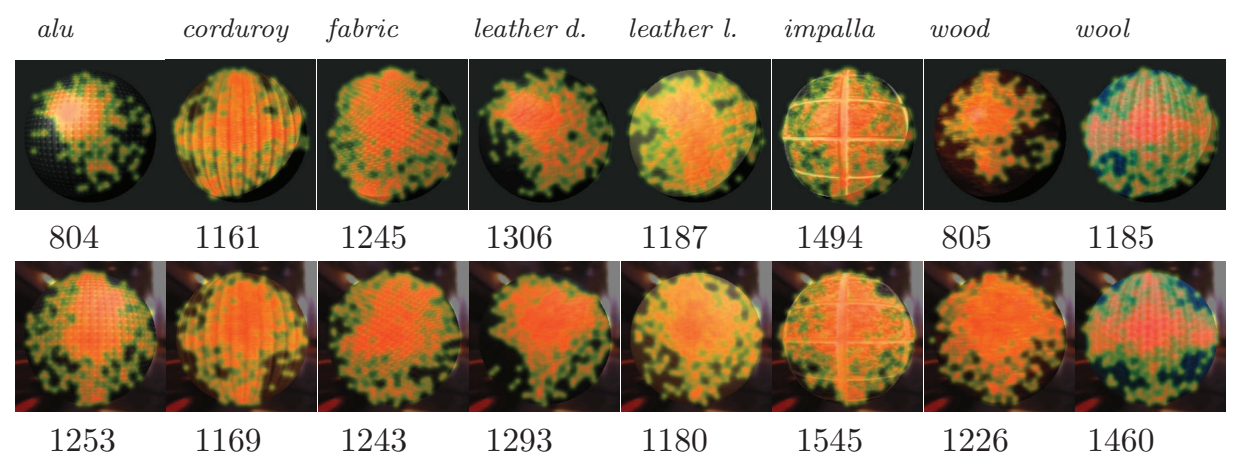

Fig. 10: BTF rendered spheres overlaid by all collected subjects fixations for pointlight and grace illuminations. Numbers of fixations for individual samples and illuminations are shown below.

First we analysed fixation duration as a function of ordinal fixation number. Fig. 11-left shows that the average fixation duration was the lowest for the first three fixations and then increased almost linearly with trial duration. This behaviour is similar to results in [Over et al. 2007] and suggests that subjects applied a coarse-tofine approach during visual search. The subjects notice within the first few fixations when the difference between spheres is more apparent, otherwise they spend more time by careful searching for a difference resulting in longer periods of fixations which increased proportionally with total length of the search. Fig. 11-right shows that the total number of fixations decreased almost exponentially and thus most responses to stimuli are given during the first twenty fixations. The decrease in number of fixations at the beginning of the trial was caused by subjects initially fixating on fixation cross in the middle of the screen before the stimulus appeared (Fig. 5-a.), i.e., outside the textured spheres and thus those fixations were ignored. This helped us to avoid possible problems with central fixation bias [Tatler 2007].

The next step was analysis of fixation locations. We employed PCA of $x$ and $y$ coordinates of selected fixations. As a result we obtained two orthogonal principal directions (eigen-vectors) and corresponding data variances along these directions (eigen-values). We visualized these data as an ellipse shaped by the obtained variances with axes oriented in the principal directions. Such an ellipse clearly represents major properties of cloud of selected fixation points. In order to ease the comparison of results of individual materials and resampling schemes we scaled the size of all ellipses using value of the largest recorded principal component. Results 

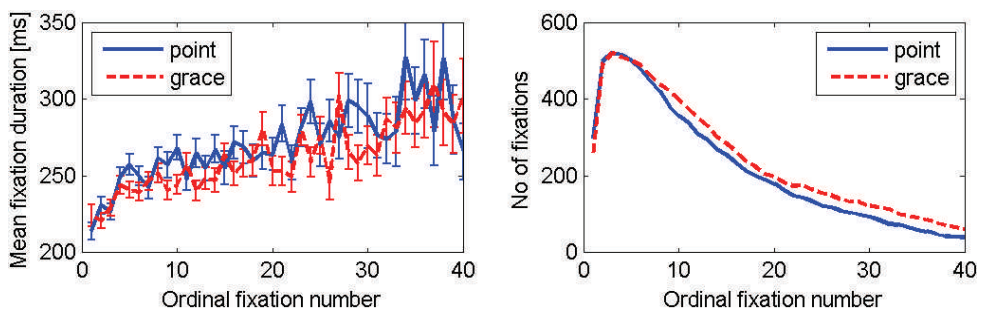

Fig. 11: Mean fixation duration (left) and number of fixations (right) as a function of ordinal fixation number for both illumination environments.

obtained results for both illuminations are shown in Fig. 12. The estimated fixation ellipses (their centers shown as red crosses) are drawn against blue unit circles representing the textured sphere in experimental stimuli. Results for point-light illumination also show the location of the ideal specular reflection using a blue cross. The figure also shows marginal fixation ellipses computed for fixations across all materials (the last column) and across all schemes (the last row respectively). The bottom-right ellipse then represents all fixations measured for a given illumination scheme.

In Fig. 12 we can see that the size and slant of fixation ellipses strongly depends on the material and resampling scheme. For instance for highly specular samples (i.e., alu, wood) is area of fixation ellipses very small which is in contrast with more diffuse materials (i.e., corduroy, fabric, wool).

Measured gaze fixations in Fig. 10 as well as the previous analysis in Fig. 12 show that majority of fixations is concentrated near center of rendered sphere. One could wonder whether is this behaviour typical and if it could be predicted automatically by some model of low level human vision system. We used Visible Difference Predictor (VDP) [Daly 1993] to assess visual differences between the rendered spheres in all experimental stimuli. Results of VDP for all stimuli, i.e., comprising different material and resampling schemes, and given the parameters of LCD screen used for psychophysical experiment, are shown in Fig. 13. Intensity of responses obtained from VDP is generally low and the highest for fabrics materials corduroy and fabric. Although VDP predicted correctly as a main differences locations of specular highlights for shiny samples illuminated by point light, for the other materials it surprisingly found places with main visual differences near edges of the spheres, i.e., for relatively high grazing angles. This is in contradiction with the measured fixations that are concentrated mostly on the center of sphere. From the results for individual resampling schemes we can conclude that VPD can predict the visual degradation relatively correctly in accordance with the results obtained from the psychophysical experiment (the highest differences found for scheme $\mathbf{C} \times \mathbf{C}$, the lowest response for scheme $\mathbf{B} \times \mathbf{8 1}$ ). On the other hand it is quite difficult to assess visual differences between resampling schemes $\mathbf{A} \times \mathbf{A}, \mathbf{B} \times \mathbf{B}, \mathbf{8 1} \times \mathbf{B}$.

If we analyse shapes of the fixation ellipses shown in Fig. 12 more closely we can spot several patterns. First is the typical slant and shape of ellipses that reflects the illumination gradient of the illumination (see Fig. 14). This suggests that locations in material structure in the direction of the highest illumination gradient were the most important aspect for subject when assessing the similarity of textures. To ver- 
ify this hypothesis in Tab. I we compared slants of the highest gradient in rendered images with estimated slants of fixation ellipses (see Fig. 12) for all tested materials. To compute main gradient reliably we used low-pass Gaussian filter to remove regular texture structures from the rendered images and used only the required overall

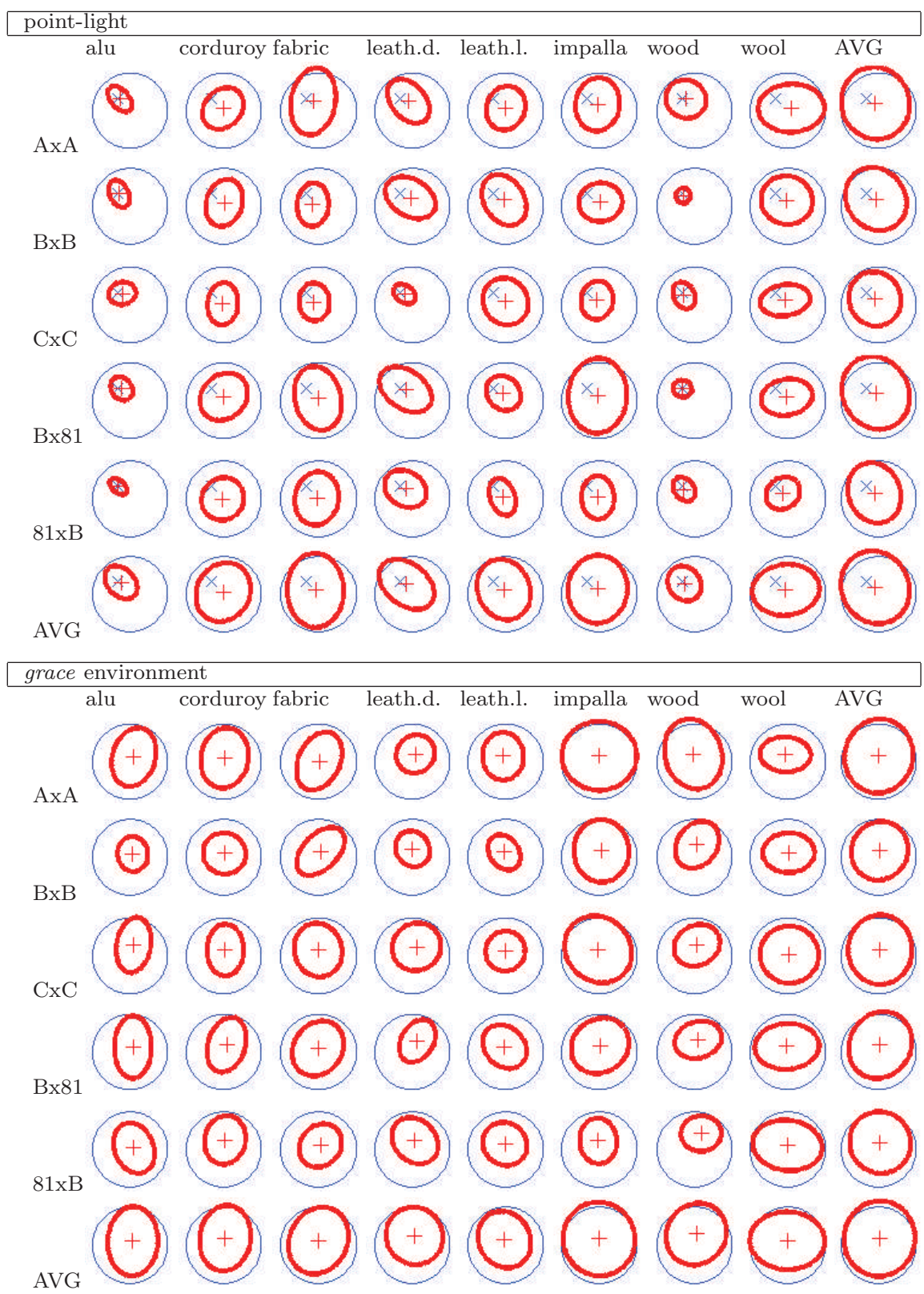

Fig. 12: Fixation ellipses for individual schemes (rows) and samples (columns) for point-light and grace environment. 
reflectance characteristics of individual materials (resembling to material BRDF). The table also includes ratios of sizes of ellipse axes. The higher is the ratio the

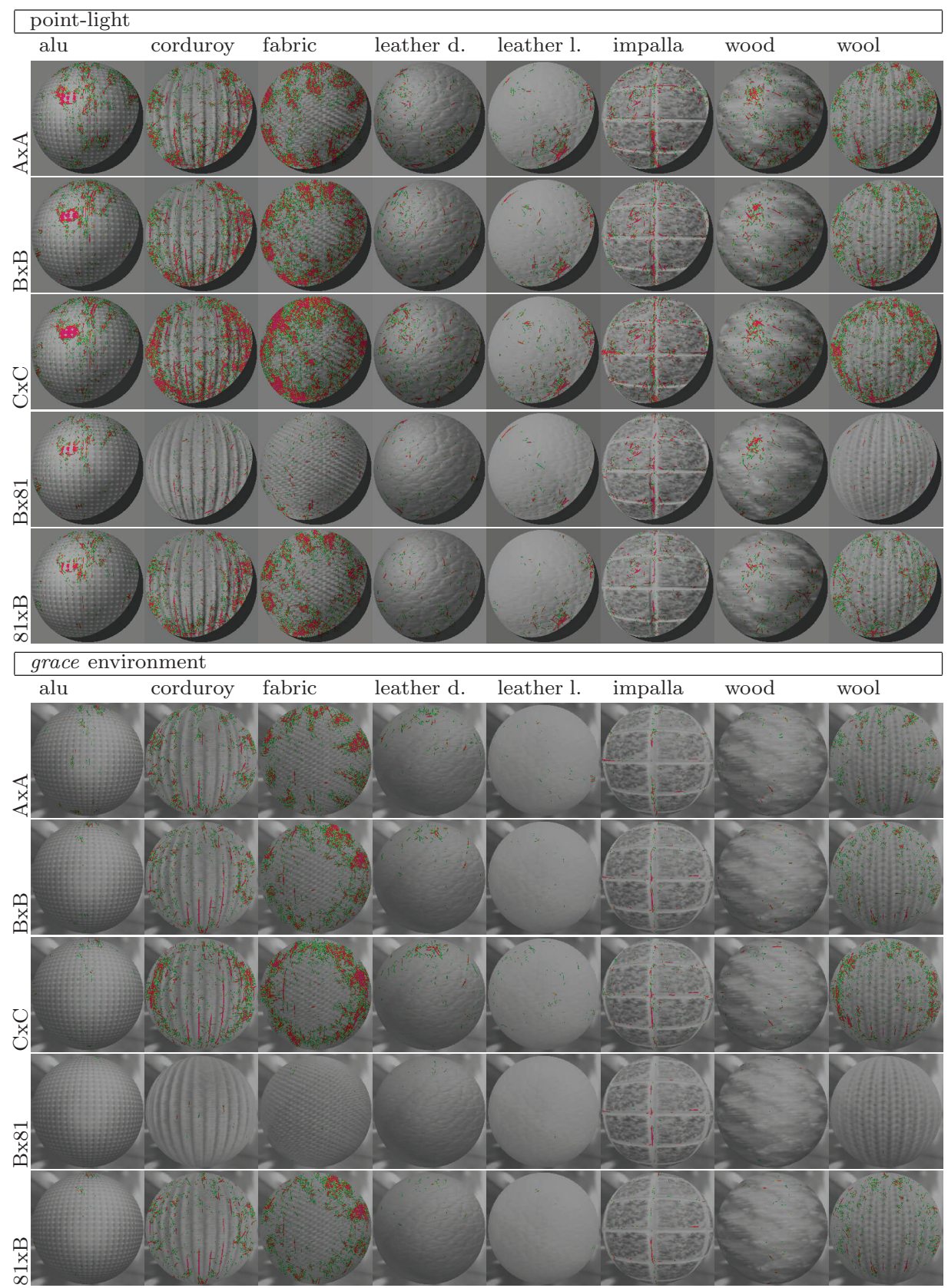

Fig. 13: Responses of visible difference predictor for tested materials and resampling schemes in point-light and grace illumination.

ACM Transactions on Applied Perception, Vol. V, No. N, Month 20YY. 
(a)

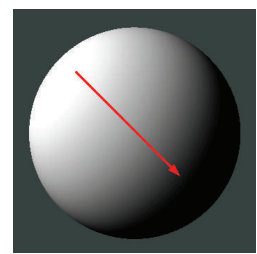

(b)

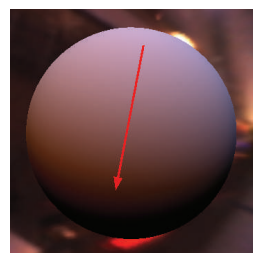

Fig. 14: Major illumination gradients for the used illumination environments: point-light and grace.

Table I: Correlation of main gradient slant in rendered images (the second and fifth columns) compared with estimated slant of fixation ellipse (the third and sixth columns) and ratio of ellipses axes sizes (the fourth and seventh columns).

\begin{tabular}{|l|ccc|ccc|}
\hline & \multicolumn{3}{|c|}{ point-light } & \multicolumn{3}{c|}{ grace environment } \\
\hline & $\begin{array}{c}\text { gradient } \\
\text { material }\end{array}$ & flant & slantion & rllipse & gradient & \multicolumn{2}{c|}{ fixation ellipse } \\
slant & slant & ratio \\
\hline alu & 137 & 139 & 2.0 & 72 & 87 & 1.8 \\
corduroy & 135 & 59 & 1.3 & 28 & 81 & 1.6 \\
fabric & 134 & 92 & 1.8 & 24 & 63 & 1.4 \\
foil01 & 132 & 142 & 2.2 & 29 & 37 & 1.1 \\
foil02 & 127 & 113 & 1.4 & 70 & 123 & 1.3 \\
impalla & 108 & 85 & 1.3 & 84 & 117 & 1.1 \\
wood & 138 & 130 & 1.4 & 22 & 46 & 1.2 \\
wool & -10 & 7 & 1.7 & 10 & -2 & 1.7 \\
\hline mean & $\mathbf{1 1 3}$ & $\mathbf{9 6}$ & 1.6 & $\mathbf{4 2}$ & $\mathbf{6 9}$ & 1.4 \\
\hline
\end{tabular}

more directionally are fixations aligned and thus the estimated value of slant angle is more significant. A reflectance data are not always consistent with computed illumination gradient. This is often the case for the anisotropic samples corduroy, fabric, impalla. However, when we computed correlation coefficient between both sets of slants for all materials we obtained quite significant values: $R=0.808$ for point-light and $R=0.865$ for grace environment. This result suggests that direction of main gradient in rendered images can effectively predict main direction of fixation cloud and thus the most probable locations of observer's fixations. As the main gradient is mainly caused by illumination gradient we can extend the previous conclusion to illumination gradient at least for majority of the tested materials.

Another typical feature is size of fixation ellipses. We have found that the area of the ellipses strongly correlates with the ease of finding a difference between textures. In other words, the less apparent the difference, the wider the search and hence more fixations were needed. If we translate this fact into the proposed comparison of different resampling schemes ones having large fixation ellipses should be the best. Similarly, a differences in materials having the largest fixation area are hard to distinguish (e.g., impalla). On the other hand materials with a salient specular highlight have small fixation areas and can be relatively easily distinguished regardless of the resampling scheme used (e.g., alu, leather dark, wood). For these materials subjects made their decision comparing only the areas of specular highlights. This is confirmed in Fig. 15 showing average Euclidean distance of mean fixation from location of ideal specular reflection. Error-bars represent twice the standard error across all resampling schemes. 


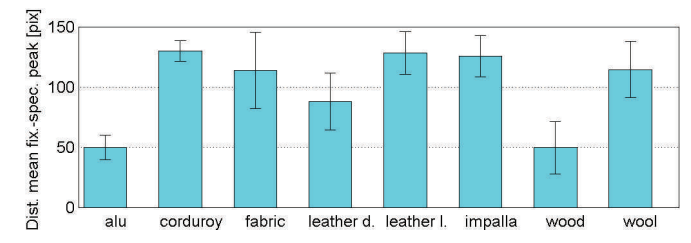

Fig. 15: Distance between specular peak and average fixation for different samples and point light illumination.

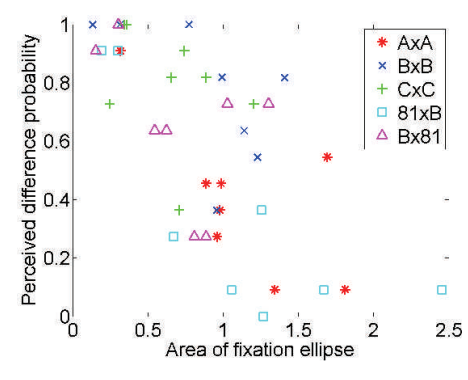

Fig. 16: Correlation of perceived difference probability and area of fixation ellipse.

We visualized data for different materials and resampling schemes in a similar manner to that described in Section 5.1. Fig. 8 shows fixation areas for resampling schemes $\mathbf{A} \times \mathbf{A}, \mathbf{B} \times \mathbf{B}$, and $\mathbf{C} \times \mathbf{C}$. Fig. 9 illustrates fixation areas for resampling schemes $\mathbf{8 1} \times \mathbf{B}, \mathbf{B} \times \mathbf{8 1}$, and $\mathbf{B} \times \mathbf{B}$. Both figures show results for point-light (a) and grace environment illumination (b). The similarity of the graphs for point-light with Fig. 15 for different materials confirms our assumption that difference in specular highlights is the most salient feature influencing subjects' assessment. Results in the figures are inversely proportional to data in Fig. 6 and Fig. 7. Generally, the higher the perceived difference in the stimuli the smaller is area of a corresponding fixation ellipse. This tendence is apparent also in Fig. 16 showing correlation of perceived difference probability obtained from subject responses and area of fixation ellipse obtained from the gaze data analysis.

To sum up this paper we have compared mean perceived differences of subjects from Section 5.1 in Fig. 17-a with areas of fixation ellipses in Fig. 17-b averaged across different materials. We can again observe that perceived difference is inversely related to area of fixation ellipse. Error-bars in graphs represent twice the standard error across all materials. Additionally, the subjects gaze fixation analysis

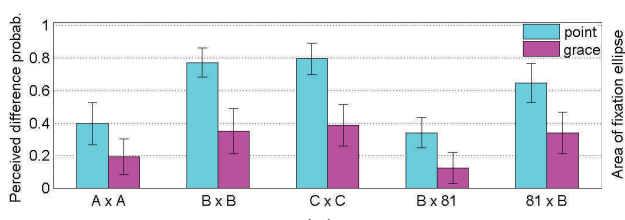

(a)

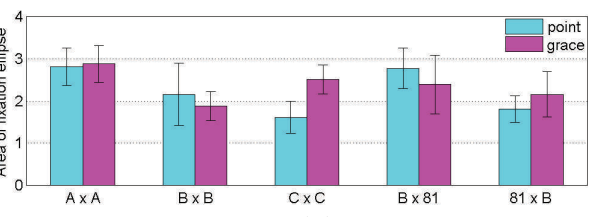

(b)

Fig. 17: Mean perceived differences (a) and fixation areas (b) for different resampling schemes.

supports our conclusion that resampling over azimuthal angle (and/or applied to illumination direction only) is the most convenient. The reason for this behaviour might be that change of elevation angle has generally much higher impact on texture brightness that the same change in azimuthal angle. This may be intuitively explained by simple Lambertial model where the reflectance value depends only on elevation angle. So the closer is the material reflectance to Lambertian model the smaller difference is between resampling schemes A and B. In Fig. 6 we can see that materials with generally diffuse appearance leather $d$., leather $l$. have similar 
values for both schemes which is not the case for other more anisotropic materials as fabrics and lacquered wood.

\section{CONCLUSIONS}

The main goal of this paper was to determine the optimal uniform resampling of view and illumination data without significant loss of their visual quality. This was achieved by means of psychophysical experiments using several resampling strategies applied on eight bidirectional texture function datasets corresponding to several natural and man-made materials. An analysis of the subjects' responses and their gaze fixations showed that optimal resampling of these data should reduce the number of samples along azimuthal and preserve original elevation view / illumination angles. Another important conclusion is that sampling of illumination direction can be significantly more sparse than sampling of view direction where the close-to-original sampling should be preserved to avoid significant blur in resampled data. Additionally, environment lighting is more convenient when resampled data are used, since the distortions introduced can be hidden by the convolution of the pixel with the underlying light pattern and low sampling is not so apparent to the observer. The gaze fixation analysis revealed that subjects prefered to observe materials in directions of illumination gradient when making their judgements.

To sum up, our results have shown that even uniform resampling of view/illumination data can often significantly reduce the size of the required dataset without introducing significant perceptible differences. This simple result may benefit many compression, modeling, or rendering methods, which use this type of massive reflectance data.

Acknowledgments: We would like to acknowledge Bonn University for providing the measured BTF samples, all the participants for help with the experiment, and anonymous reviewers for helpful comments. J. Filip was supported by the EC Marie-Curie IEF No.41358, the EPSRC SMI grants GR/S12395/01 and EP/F02553X/1, the GAČR grant 102/08/0593, and grant MŠMT 1M0572 (DAR).

\section{REFERENCES}

Carr, J., Beatson, R., Cherrie, J., Mitchell, T., Fright, W., McCallum, B., and Evans, T. 2001. Reconstruction and representation of $3 \mathrm{D}$ objects with radial basis functions. $A C M$ SIGGRAPH 2001, 67-76.

Cochran, W. G. 1950. The comparison of percentages in matched samples. Biometrika, 37, 256-266.

DALY, S. 1993. The visible differences predictor: an algorithm for the assesment of image fidelity. Digital Images and Human Vision, 179-206.

Dana, K., van Ginneken, B., Nayar, S., And Koenderink, J. 1999. Reflectance and texture of real-world surfaces. ACM Transactions on Graphics 18, 1, 1-34.

Deng, Z., Lewis, J. P., And Neumann, U. 2005. Automated eye motion using texture synthesis. IEEE Computer Graphics and Applications 25, 2, 24-30.

Duchowski, A., Cournia, N., And Murphy, H. 2004. Gaze-contingent displays: A review. Gaze-Contingent Displays: A Review 6, 7, 621-634.

Duchowski, A. T. 2002. A breadth-first survey of eye-tracking applications. Behav Res Methods Instrum Comput 34, 4 (November), 455-470.

Elhelw, M. A., Nicolaou, M., Chung, J. A., Yang, G.-Z., And Atkins, M. S. 2008. A 
gaze-based study for investigating the perception of visual realism in simulated scenes. $A C M$ Transactions on Applied Perception 5, 1.

Filip, J., AND HAindL, M. 2009. Bidirectional texture function modeling: A state of the art survey. To appear in IEEE Transactions on Pattern Analysis and Machine Intelligence.

Filip, J., Chantler, M., Green, P., And Haindl, M. 2008. A psychophysically validated metric for bidirectional texture data reduction. ACM Transactions on Graphics 27, 5 (December), 138.

Fleming, R. W., Dror, R. O., And Adelson, E. H. 2003. Real-world illumination and perception of surface reflectance properties. In Journal of Vision, vol. 3, 347-368.

Havran, V., Smyk, M., Krawczyk, G., Myszkowski, K., and Seidel, H.-P. 2005. Interactive system for dynamic scene lighting using captured video environment maps. In Eurographics Symposium on Rendering, 31-42,311.

Kautz, J., Boulos, S., And Durand, F. 2007. Interactive editing and modelling of bidirectional texture functions. ACM Trans. Graph. 26, 3, 53.

Lawson, R., Bülthoff, H., And Dumbell, S. 2003. Interactions between view changes and shape changes in picture - picture matching. Perception 34, 12, 1465-1498.

Lee, S. P., Badler, J. B., And Badler, N. I. 2002. Eyes alive. ACM Transactions on Graphics 21, 3 (July), 637-644.

Matusik, W., Pfister, H.P. Brand, M., And McMillan, L. 2003. A data-driven reflectance model. ACM Transaction on Graphics 22, 3, 759-769.

Meseth, J., Müller, G., Klein, R., Röder, F., And Arnold, M. 2006. Verification of rendering quality from measured BTFs. In Third Symposium on Applied Perception in Graphics and Visualization, 127-134.

Müller, G., Meseth, J., Sattler, M., Sarlette, R., And Klein, R. 2005. Acquisition, synthesis and rendering of bidirectional texture functions. Computer Graphics Forum 24, 1 (March), 83-110.

Nicodemus, F., J.C., R., Hsia, J., Ginsburg, I., And Limperis, T. 1977. Geometrical considerations and nomenclature for reflectance. NBS Monograph 160, National Bureau of Standards, U.S. Dept. of Commerce, Washington, D. C., 1-52.

Over, E., Hooge, I., Vlaskamp, B., and Erkelens, C. 2007. Coarse-to-fine eye movement strategy in visual search. Vision Research 47, 17, 2272-2280.

Pellacini, F., And Lawrence, J. 2007. Appwand: editing measured materials using appearancedriven optimization. ACM Transactions on Graphics 26, 3, 54:1-54:10.

Pellacini, F., Ferwerda, J., And GreenberG, D. 2000. Toward a psychophysically-based light reflection model for image synthesis. In 27 th International Conference on computer Graphics and Interactive Techniques, 55-64.

Pomplun, M. 2006. Saccadic selectivity in complex visual search displays. Vision Research 46 .

Ramanarayanan, G., Ferwerda, J., Walter, B., And Bala, K. 2007. Visual equivalence: towards a new standard for image fidelity. ACM Transactions on Graphics 26, 3, 76:1-76:10.

Sundstedt, V., Stavrakis, E., Wimmer, M., and Reinhard, E. 2008. A psychophysical study of fixation behavior in a computer game. In $A P G V$ '08: Proceedings of the 5th symposium on Applied perception in graphics and visualization, ACM, 43-50.

TAtLER, B. W. 2007. The central fixation bias in scene viewing: Selecting an optimal viewing position independently of motor biases and image feature distributions. Journal of Vision 7 , 14 (November), 1-17.

TE PAS, S., AND Pont, S. 2005. A comparison of material and illumination discrimination performance for real rough, real smooth and computer generated smooth spheres. In $A P G V$ '05: 2nd Symposium on Applied perception in graphics and visualization, 57-58.

TE PAS, S., AND PONT, S. 2005. Estimations of light-source direction depend critically on material BRDFs. Perception, ECVP Abstract Supplement 34, 212.

Manuscript received February 2009; revised May 2009.

ACM Transactions on Applied Perception, Vol. V, No. N, Month 20 YY. 